\title{
Capacities and Games on Lattices: A Survey of Results
}

\author{
Michel GRABISCH \\ Université Paris I - Panthéon-Sorbonne \\ Michel.Grabisch@lip6.fr
}

November 16, 2007

\begin{abstract}
We provide a survey of recent developments about capacities (or fuzzy measures) and ccoperative games in characteristic form, when they are defined on more general structures than the usual power set of the universal set, namely lattices. In a first part, we give various possible interpretations and applications of these general concepts, and then we elaborate about the possible definitions of usual tools in these theories, such as the Choquet integral, the Möbius transform, and the Shapley value.
\end{abstract}

Keywords: capacity, fuzzy measure, game, lattice, Choquet integral, Shapley value

\section{Introduction}

Among the recent advances in capacity (or fuzzy measures) and cooperative game theory, a notable fact is the emergence of new notions of capacities and games which are defined on more general structures than the usual Boolean lattice of the subsets of the universal set. Apart of the mathematical interest brought by such works, the main motivation lies in an attempt to model the real world in a more accurate way.

As it is often the case with generalizations, the main difficulty is to find the right definitions for the usual tools and concepts used in the theory. Concerning capacity theory, fundamental concepts are the Choquet integral and the Möbius transform, while for cooperative game theory, the Shapley value and the core are important notions.

Our aim is to provide a survey of recent advances along these lines. We will see that, although the generalization of the Möbius transform and Choquet integral do not raise particular difficulties, a proper definition of the Shapley value is much more a topic of discussion. We will address also the case of bipolar structures, and show that these structures cannot be reduced to a classical lattice structure, although they can be isomorphically mapped to lattices.

In all our discussion, we consider the universal set to be finite. We denote it by $N$, and $|N|=n$. 


\section{Capacities, fuzzy measures, games and the like}

Definition $1 A$ capacity on $N$ is a set function $\mu: 2^{N} \rightarrow \mathbb{R}_{+}$such that $\mu(\emptyset)=0$, and $A \subseteq B \subseteq N$ implies $\mu(A) \leq \mu(B)$. This last property is called monotonicity.

Although the notion of capacity is usually attributed into our field to Choquet [10], capacities arised from the problem of electric distribution and they have been studied by many mathematicians before Choquet. We could cite for example Florin Vasilesco [52], who surveyed the notion of capacities before 1937, and Henri Cartan [8], who studied the capacities of compact sets.

A capacity is normalized if $\mu(N)=1$. In 1974, Sugeno proposed a similar notion (up to some condition of continuity), which he called fuzzy measures 48. Other names which are commonly used are nonadditive measures (Denneberg [13]), and monotonic measures.

Definition $2 A$ transferable utility game in characteristic form or for simplicity game, is a set function $v: 2^{N} \rightarrow \mathbb{R}$ such that $v(\emptyset)=0$.

The above definition is the central concept of cooperative game theory (see, e.g., 14, 11, 3, 44). The only difference between games and capacities is that monotonicity is dropped for the former. Hence monotonic games coincide with capacities, and non monotonic fuzzy measures, a term which is sometimes used, coincide with games. In the sequel, notation $v$ implicitely designates a game, while $\mu$ refers to a capacity.

Given a subset $A \subseteq N$, the precise meaning of the quantity $\mu(A)$ or $v(A)$ depends on the kind of the intended application or domain, in fact essentially what the universal set $N$ is supposed to represent.

- $N$ is the set of states of nature. Then $A \subseteq N$ is an event, and $\mu(A)$ is the degree of certainty, belief, etc., that $A$ contains the true state of the world. We are here in decision under uncertainty or under risk.

- $N$ is the set of criteria, or attributes, or sources. Then $A \subseteq N$ is a group of criteria (or attributes, sources), and $\mu(A)$ is the degree of importance of $A$ for making decision. Corresponding domains are multicriteria decision making, multiattribute utility theory, multiattribute classification, data fusion, etc. In the framework of multicriteria decision making, it is possible to give a more precise definition for $\mu(A)$ : it is the overall score of an alternative having score $=1$ (maximal) for all criteria in $A$, and 0 (minimal) for other criteria [30, 39. This kind of interpretation can be carried on other domains as well.

- $N=$ set of voters. Then $A \subseteq N$ is called a coalition, and $v(A)=1$ iff the bill passes when coalition $A$ votes in favor of the bill, and $v(A)=0$ else.

- $N=$ set of political parties. Then $A \subseteq N$ is called a coalition, and $v(A)=1$ iff the coalition of parties wins the election, and $v(A)=0$ else. These two last examples are a subdomain of cooperative game theory, called voting games. 
- $N=$ set of players, agents, companies, etc. Then $A \subseteq N$ is also called a coalition, and $v(A)$ is the worth (payoff, income, etc.) won by $A$ if all players in $A$ agree to cooperate, and the other ones do not. The concerned domain is cooperative game theory.

\section{Generalizations of games and capacities}

\subsection{Motivations}

A first question is:

Why do we need generalizations of classical games and capacities?

The answer to this question is simply that we need them in order to model reality in a more accurate way. Let us elaborate on this, and distinguish several cases.

- A first situation is that some subsets of $N$ may be not meaningful, so that the structure is no more the Boolean lattice $2^{N}$ of all subsets of $N$, but a subcollection of it. More specifically, when $N$ is the set of states of nature, some events may be not observable or not meaningful. Note that in probability theory, it is the usage to define probabilities on algebras (families of subsets closed under unions and complement), not on the whole power set. In the case of political parties, it means that some coalitions of parties are unlikely to occur, or even impossible (coalitions mixing left and right parties). When $N$ is the set of voters, it means that some voting situations (i.e., the set of voters voting in favor) are unlikely to occur or impossible. Lastly, when $N$ is the set of players in a general sense, it may happen that some coalitions are infeasible, for some reasons depending on the precise meaning attached to players (e.g., competitive companies for which it is impossible to cooperate).

- A second possibility is that subsets of $N$ may be not "black and white", which means that the membership of an element to $N$ may be not simply resume to a matter of member or nonmember. This is the case with multicriteria decision making when underlying scales are bipolar, i.e., a central value exists on each scale, which is a demarcation between values considered as "good", and values considered as "bad", the central value being neutral. When building the model, we must then distinguish for a given alternative criteria which have a good value, from those which have a bad value (or a neutral one). In voting games, it is convenient to consider that players may also abstain, hence each voter has three possibilities, so that giving only the set of voters voting in favor is not enough to describe the voting situation (ternary voting games). When $N$ is the set of players, one may consider that each player can play at different levels of participation, ranging from no participation to full participation. If there is a finite number of such participation levels, it corresponds to multichoice games, when a degree of participation is defined on $[0,1]$, it corresponds to fuzzy games.

- A last possibility is that, after all, elements of interest may be not subsets of $N$. Global games work on partitions of players, not on coalitions, while 
games in partition function form and global coalitional games work on the set of partitions and coalitions together.

\subsection{Examples of generalized games}

Let us introduce main examples of games defined on more general structures.

Games on convex geometries (Bilbao 1998) [2, 1, 3]: a collection $\mathcal{L}$ of subsets of $N$ is a convex geometry if it contains the empty set, is closed under intersection, and $S \in \mathcal{L}, S \neq N$ implies that it exists $j \in N \backslash S$ such that $S \cup j \in \mathcal{L}$. Then, $v: \mathcal{L} \rightarrow \mathcal{R}$ is a game on convex geometry $\mathcal{L}$ if $v(\emptyset)=0$. Convex geometries are dual of antimatroids (see, e.g., [38]), and Bilbao studied also games defined on matroids [3], which are an abstraction of independent systems (see again $[38]$ ).

Games with precedence constraints (Faigle 1989) [16, 17]: $N$ being the set of players, let us define a partially ordered set $P:=(N, \leq)$, where $\leq$ is a relation of precedence among players: $i \leq j$ if the presence of $j$ enforces the presence of $i$ in any coalition $S \subseteq N$. Hence, a valid coalition of $P$ is a subset $S$ of $N$ such that $i \in S$ and $j \leq i$ entails $j \in S$.

Ternary voting games (Felsenthal and Machover 1997) [18]: a ternary voting game is a voting game where each voter $i \in N$ may vote in favor, against or abstain. Hence, a voting situation is denoted $(A, B)$, where $A$ is the set of voters voting in favor, and $B$ those voting against. Introducing the notation

$$
\mathcal{Q}(N):=\{(A, B) \mid A, B \subseteq N, A \cap B=\emptyset\}
$$

which represents the set of all voting situations, a ternary voting game is a function $v: \mathcal{Q}(N) \rightarrow\{-1,1\} \cdot v(A, B)=1$ iff the bill passes in voting situation $(A, B), v(A, B)=-1$ iff the bill is rejected.

Another way of denoting a situation $(A, B)$ is to use a vector notation $x \in$ $\{-1,0,1\}^{n}$ defined as follows:

$$
(A, B) \in \mathcal{Q}(N) \cong x \in\{-1,0,1\}^{n}, \text { with } x_{i}= \begin{cases}1, & \text { if } i \in A \\ -1, & \text { if } i \in B \\ 0, & \text { else. }\end{cases}
$$

Hence $\mathcal{Q}(N) \cong\{-1,0,1\}^{n} \cong 3^{N}$.

Bi-cooperative games (Bilbao 2000) [3]: they can be seen as a generalization of ternary voting games, like voting games are generalized to (classical) cooperative games. In such games, each player $i \in N$ may play as a defender, a defeater, or does not participate. A bi-coalition $(A, B) \in \mathcal{Q}(N)$ represents a situation where $A$ is the defending coalition, and $B$ the opponent coalition. A bi-cooperatice game is a function $v: \mathcal{Q}(N) \rightarrow \mathbb{R}$ such that $v(\emptyset, \emptyset)=0 . v(A, B)$ is the payoff of the game in situation $(A, B)$. 
Multichoice games (Hsiao and Raghavan 1993) [35]: each player $i \in N$ has at disposal a totally ordered set of levels of participation labelled $0,1, \ldots, m$, where 0 indicates no participation, and $m$ full participation. A coalition is replaced by a profile of participation $x \in\{0,1, \ldots, m\}^{n}$, where $x_{i}$ is the level of participation of player $i$. A multichoice game is a function $v:\{0,1, \ldots, m\}^{n} \rightarrow$ $\mathbb{R}$ such that $v(0, \ldots, 0)=0$. The quantity $v(x)$ is the payoff of the game for profile $x$.

Fuzzy games (Aubin, 1981) [1]: each player has a membership degree in a coalition, considered as a fuzzy set. It can be seen as a multichoice game with a continuum of level of participations. Fuzzy games have been studied by Butnariu and Klement [7, and more recently by Branzei and Tijs [6, 50].

Global games (Gilboa and Lehrer 1991) [21]: let us consider the set of partitions of $N$, which we denote by $\Pi(N)$. When endowed with the relation of coarseness (i.e., a partition $\mathcal{P}$ is coarser than a partition $\mathcal{P}^{\prime}$ if any set of $\mathcal{P}$ is a superset of some set of $\mathcal{P}^{\prime}$ ), the set of partition is a lattice (nondistributive, but geometric). Figure 3.2 shows the lattice of partitions of $\{1,2,3,4\}$. A global game is a function $v: \Pi(N) \rightarrow \mathbb{R}$.

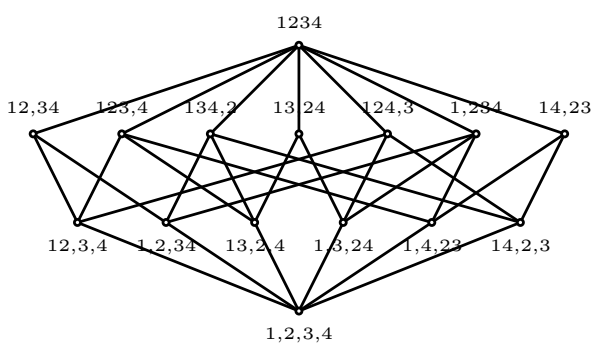

Figure 1: The lattice of partitions of $\{1,2,3,4\}$

Games in partition function form (Thrall and Lucas 1963) 49]: in these games, the worth of a coalition $A$ depends on the other coalitions which are formed, supposing the set of formed coalitions is a partition of $N$. For a given partition $\mathcal{P} \in \Pi(N)$, a quantity $v(S, \mathcal{P})$ is defined for any $S \in \mathcal{P}$.

\subsection{Examples of generalized capacities}

There is much less examples in this category. Here are the few examples we are aware of.

Bi-capacities (Grabisch and Labreuche 2002) [25, 25]: they have been introduced in the field of multicriteria decision making. Let $N$ be the set of criteria. Each criterion $i \in N$ is defined on a bipolar scale: a neutral level exists (most often the value 0 is taken as neutral level), such that values above it are felt as "good", and values below it are felt as "bad" by the decision maker. 
Hence, 3 reference levels are needed to describe the DM's preferences: the satisfactory level (usually the value 1), the neutral level (0), and the inacceptable level (usually taken as the value -1 ). Any combination of the 3 levels is called a ternary alternative, denoted by $(A, B): A$ is the set of satisfied criteria, and $B$ the set of unsatisfied criteria. Hence, $\mathcal{Q}(N)$ is the set of ternary alternatives.

A bi-capacity is a function $v: \mathcal{Q}(N) \rightarrow \mathbb{R}$ such that $v(\emptyset, \emptyset)=0$, and $A \subseteq$ $B$ implies $v(A, \cdot) \leq v(B, \cdot), v(\cdot, A) \geq v(\cdot, B)$. If normalization applies then $v(N, \emptyset)=1$ and $v(\emptyset, N)=-1$.

Although bi-cooperative games and bi-capacities were proposed independently and in different domains, formally bi-capacities are monotonic normalized bi-cooperative games.

$k$-ary capacities (Grabisch and Labreuche 2003) [24]: instead of considering 3 reference levels as for bi-capacities, $k+1$ reference levels are considered on each criterion, their meaning depending on the application considered. $k$-ary capacities correspond in fact to monotonic multichoice games.

\section{Games and capacities on lattices}

All previous examples of games and capacities are particular cases of games and capacities on lattices.

Definition 3 Let $L$ be a set and $\leq$ a partial order (antisymmetric and transitive) on $L .(L, \leq)$ is said to be a lattice if for any $x, y \in L$, the least upper bound $x \vee y$ and the greatest lower bound $x \wedge y$ always exist. $\top$ and $\perp$ are the greatest and least elements of $L$, if they exist.

Definition 4 Let $(L, \leq)$ be a lattice.

(i) $v: L \rightarrow \mathbb{R}$ is a game on lattice $L$ if $v(\perp)=0$.

(ii) $\mu: L \rightarrow \mathbb{R}_{+}$is a capacity on lattice $L$ if it is a monotonic game, i.e. $x \leq y$ implies $v(x) \leq v(y)$ (isotone or order-preserving mapping from $(L, \leq)$ to $(\mathbb{R}, \leq))$.

We denote by $\mathcal{G}(L)$ the set of games on $L$.

\subsection{Some useful facts on lattices}

We give in this section some basic results and definitions on lattices which are useful for the sequel (for a good introduction to the topic, see [12]).

For $x, y \in L$, we say that $x$ covers $y$ (denoted $x \succ y$ ) if $x>y$ and there is no $z$ such that $x>z>y$. The lattice is distributive if $\vee, \wedge$ obey distributivity.

An element $j \in L$ is join-irreducible if it is not the bottom element and it cannot be expressed as a supremum of other elements. Equivalently $j$ is joinirreducible if it covers only one element. Join-irreducible elements covering $\perp$ are called atoms, and the lattice is atomistic if all join-irreducible elements are atoms. The set of all join-irreducible elements of $L$ is denoted $\mathcal{J}(L)$. 
An important property is that in a distributive lattice, any element $x$ can be written as an irredundant supremum of join-irreducible elements in a unique way (Birkhoff theorem):

$$
x=\bigvee_{i \in J} i, \quad \text { for some } J \subseteq \mathcal{J}(L)
$$

$P \subseteq L$ is a downset or ideal if $y \leq x$ and $x \in P$ imply $y \in P$. Remarking that in a distributive lattice one can always write $x=\bigvee_{i \in \mathcal{J}(L) \mid i<x} i$, Birkhoff's theorem can be rephrased as follows: any distributive lattice is isomorphic to the lattice of all downsets of $\mathcal{J}(L)$.

In a finite setting, Boolean lattices are of the type $2^{N}$ for some set $N$, i.e. they are isomorphic to the lattice of subsets of some set, ordered by inclusion. Boolean lattices are atomistic, and atoms corresponds to singletons. A linear lattice is such that $\leq$ is a total order. All elements are join-irreducible, except $\perp$.

Given lattices $\left(L_{1}, \leq_{1}\right), \ldots,\left(L_{n}, \leq_{n}\right)$, the product lattice $L=L_{1} \times \cdots \times L_{n}$ is endowed with the product order $\leq$ of $\leq_{1}, \ldots, \leq_{n}$ in the usual sense. Elements of $x$ can be written in their vector form $\left(x_{1}, \ldots, x_{n}\right)$. We use the notation $\left(x_{A}, y_{-A}\right)$ to indicate a vector $z$ such that $z_{i}=x_{i}$ if $i \in A$, and $z_{i}=y_{i}$ otherwise. Similarly $L_{-i}$ denotes $\prod_{j \neq i} L_{j}$, while $L_{K}:=\prod_{j \in K} L_{j}$. All join-irreducible elements of $L$ are of the form $\left(\perp_{1}, \ldots, \perp_{j-1}, i_{0}, \perp_{j+1}, \ldots, \perp_{n}\right)$, for some $j$ and some joinirreducible element $i_{0}$ of $L_{j}$.

A vertex of $L$ is any element whose components are either top or bottom. We denote $\Gamma(L)$ the set of vertices of $L$. Note that $\Gamma(L)=L$ iff $L$ is Boolean.

\subsection{Games on product lattices}

We focus from now on on a specific type of game on lattice, where the lattice is a product of distributive lattices. The motivation for such an approach will be given below.

We consider $L:=L_{1} \times \cdots \times L_{n}$, where $L_{1}, \ldots, L_{n}$ are finite distributive lattices. Each lattice $L_{i}$ represents the (partially) ordered set of actions, choices, levels of participation of player $i$ to the game. Each lattice may be different.

Let us show that most of previous examples can be casted into this framework. If $L_{i}:=\{\perp, \top\}, \forall i \in N$, then we get classical games on $2^{N}$. If $L_{i}=$ $\{0,1,2\}, \forall i \in N$ we obtain bi-cooperative games (however, see Sec. 10), and ternary voting games on $3^{N}$. If $L_{i}=\{0,1, \ldots, m\}, \forall i \in N$ we obtain multichoices games on $(m+1)^{N}$

One may wonder how the set $L_{i}$ of all possible actions of player $i$ can be obtained, and why it should be distributive. We consider that each player $i \in N$ has at his disposal a set of elementary or pure actions $j_{1}, \ldots, j_{n_{i}}$. These elementary actions form a partially ordered set $\left(\mathcal{J}_{i}, \leq\right)$, but not necessarily a lattice. Then the set $\left(\mathcal{O}\left(\mathcal{J}_{i}\right), \subseteq\right)$ (i.e. the set of downsets) is a distributive lattice denoted $L_{i}$, whose join-irreducible elements precisely correspond to the elementary actions, by Birkhoff's theorem.

For example, assume that players are gardeners who take care of some garden or park. Elementary actions are watering $(\mathrm{W})$, light weeding (LW), careful weeding $(\mathrm{CW})$, and pruning $(\mathrm{P})$. All these actions are benefic for the garden and clearly $\mathrm{LW}<\mathrm{CW}$, but otherwise actions seem to be incomparable. They form the following partially ordered set: 


\section{$\circ \stackrel{\circ}{\circ}$}

which in turn form the following lattice of possible actions:

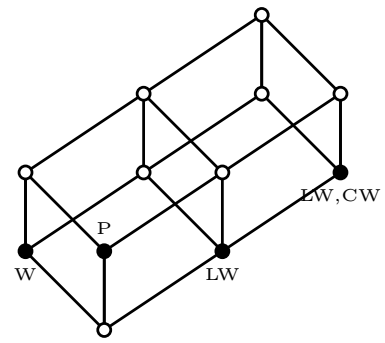

Let us give now an equivalent view of games on lattices, which is due to Faigle and Kern [17, namely games with precedence constraints. We recall that a valid coalition of $P$ is a subset $S$ of $N$ such that $i \in S$ and $j \leq i$ entails $j \in S$. Hence, the collection $\mathcal{C}(P)$ of all valid coalitions of $P$ is the collection of all downsets (ideals) of $P$. It is known that the collection of downsets of a poset is a distributive lattice. Take for example $N=\{a, b, c, d\}$, and $a \leq b, c \leq$ $b, c \leq d$ as a precedence order (Fig. 1.2). Let us show that we can recover
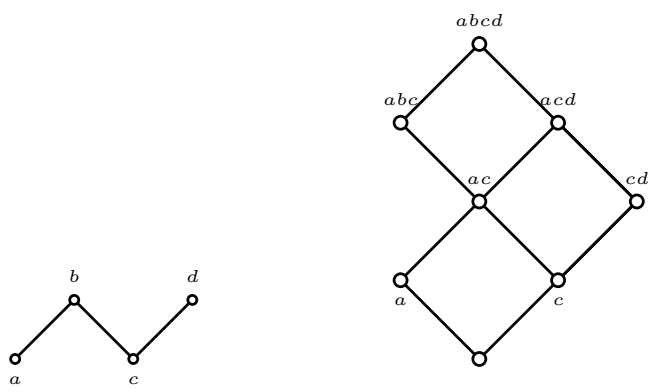

Figure 2: Exemple of precedence order (left) and the corresponding set of valid coalitions (right)

our situation, considering that $N$ is the set of players, and for each $i \in N$, let $\mathcal{J}_{i}:=\left\{j_{1}, \ldots, j_{n_{i}}\right\}$ be the set of elementary actions of player $i$. We know from the above that $L_{i}=\mathcal{O}\left(\mathcal{J}_{i}\right)$ for all $i$. We introduce now the set of virtual players

$$
N^{\prime}:=\bigcup_{i \in N} \mathcal{J}_{i}
$$

equipped with the partial order $\leq$ induced by the partial orders on each $\mathcal{J}_{i}$. Then valid coalitions of $\left(N^{\prime}, \leq\right)$ in the sense of Faigle and Kern correspond bijectively to elements of $\mathcal{O}\left(\mathcal{J}_{1}\right) \times \cdots \times \mathcal{O}\left(\mathcal{J}_{n}\right)=L_{1} \times \cdots \times L_{n}$.

\subsection{Roadmap}

This general framework being established, we should re-build usual tools from game theory and capacity theory for the general case of lattice structure. The following concepts lie among the most useful ones: 
- the Choquet integral (capacity theory)

- the Möbius transform (capacity theory), otherwise called dividends (game theory); unanimity games

- the Shapley value (game theory, capacity theory)

- the core (game theory, capacity theory)

- the entropy (probability theory, hence capacity theory).

In the sequel we provide a survey of results on these topics.

\section{The Choquet integral for bi-capacities}

Let $f: N \rightarrow[0,1]$ and a capacity $\mu$. We denote for simplicity $f(i)$ by $f_{i}, i \in N$. The Choquet integral [10 of $f$ w.r.t. $\mu$ is defined by:

$$
\int f d \mu:=\sum_{i=1}^{n}\left[f_{(i)}-f_{(i-1)}\right] \mu\left(A_{(i)}\right)
$$

with $0=: f_{(0)} \leq f_{(1)} \leq \cdots \leq f_{(n)}$ and $A_{(i)}:=\{(i), \ldots,(n)\}$, i.e., we have applied a permutation on $N$ such that $f$ becomes non decreasing. The canonical polyhedra of $[0,1]^{n}$ are defined by $\left\{x \in[0,1]^{n} \mid x_{\sigma(1)} \leq \cdots \leq x_{\sigma(n)}\right\}$, for some permutation $\sigma$ on $N$. Clearly, these canonical polyhedra partition the set of functions from $N$ to $[0,1]$ into simplices where the same "weights" $\mu()$ are used in (5).

We recall the following result.

Proposition 1 Let $F$ be a function on $[0,1]^{n}$, which is known only on the vertices of the hypercube, and let us find a simplest linear interpolation to determine $F$ entirely:

$$
F(x)=\sum_{A \subseteq N \mid\left(1_{A}, 0_{A^{c}}\right) \in \mathcal{V}(x)}\left[\alpha_{0}(A)+\sum_{i=1}^{n} \alpha_{i}(A) x_{i}\right] F\left(1_{A}, 0_{A^{c}}\right),
$$

where $\mathcal{V}(x)$ is the set of vertices used for the linear interpolation of $x$, and $\alpha_{i}(A) \in \mathbb{R}, i=0, \ldots, n, \forall A \in \mathcal{V}(x)$. Moreover, we impose that $\operatorname{conv}(\mathcal{V}(x))$ contains $x$, and any $x \in[0,1]^{n}$ should belong to a unique polyhedron (except for common facets), with continuity ensured (triangulation of $\left.[0,1]^{n}\right)$.

Then the unique linear interpolation with no constant terms is the Choquet integral, and the triangulation is obtained by the canonical polyhedra.

Lovász [40], considering the problem of extending the domain of pseudoBoolean functions to $[0,1]^{n}$ in a linear way (for this extension problem, see also Singer 47 ), incidentally discovered the formula of the Choquet integral. The fact that the so-called Lovász extension was the Choquet integral was remarked by Marichal [41]. The above result of uniqueness can be found in [28].

Let us apply the same interpolative approach to the case of bi-capacities. The main idea is that for a given point $x \in[-1,1]^{n}$, it suffices to go back into the positive quadrant $[0,1]^{n}$ by taking the absolute value $|x|$, and there to apply the interpolation formula (classical Choquet integral), but using vertices of the original quadrant containing $x$. This leads to the following definition. 
Definition 5 Let $v$ be a bi-capacity and $f$ be a real-valued function on $N$. The (general) Choquet integral of $f$ w.r.t $v$ is given by

$$
\int f d v:=\int|f| d \nu_{N_{f}^{+}}
$$

where $\nu_{N_{f}^{+}}$is a game on $N$ defined by

$$
\nu_{N_{f}^{+}}(C):=v\left(C \cap N_{f}^{+}, C \cap N_{f}^{-}\right),
$$

and $N_{f}^{+}:=\left\{i \in N \mid f_{i} \geq 0\right\}, N_{f}^{-}=N \backslash N_{f}^{+}$.

A similar construction can be done for $k$-ary capacities [24].

\section{The Möbius transform}

Following the general definition of Rota [45] (see also [5, p. 102]), we have readily a definition for any game defined on any lattice, or even for games defined on any partially ordered set, provided it is locally finite (i.e., any interval is finite) and with a bottom element. Let $v$ be a game on $(L, \leq)$, the Möbius transform of $v$ is the function $m: L \longrightarrow \mathbb{R}$ solution of the equation:

$$
v(x)=\sum_{y \leq x} m(y) .
$$

The expression of $m$ is obtained through the Möbius function $\mu$ by:

$$
m(x)=\sum_{y \leq x} \mu(y, x) f(y)
$$

where $\mu$ is a function on $L^{2}$ defined inductively by

$$
\mu(x, y)= \begin{cases}1, & \text { if } x=y \\ -\sum_{x \leq t<y} \mu(x, t), & \text { if } x<y \\ 0, & \text { otherwise. }\end{cases}
$$

Note that $\mu$ depends only on the structure of $(L, \leq)$.

\section{The Shapley value}

\subsection{The classical case}

The Shapley value, or more generally the notion of value, is one of the most important concept in cooperative game theory. A value or solution concept is any function $\phi: \mathcal{G}\left(2^{N}\right) \longrightarrow \mathbb{R}^{N}$, which represents an imputation of income to each player, supposing that all players will join the grand coalition $N$, so that the amount $v(N)$ has to be shared among players. The value is efficient if $\sum_{i \in N} \phi_{i}(v)=v(N)$. Among other properties or axioms values should satisfy, the following ones are classical.

- linearity (l): $\phi$ is linear over $\mathcal{G}\left(2^{N}\right)$. 
- dummy axiom (d): if $i$ is dummy for $v$, then $\phi^{v}(i)=v(i)$.

- null axiom (n): if $i$ is null for $v$, then $\phi^{v}(i)=0$.

- symmetry (s): $\phi$ does not depend on the labelling of the players.

A player $i$ is dummy if $v(S \cup i)=v(S)+v(i)$ for any $S \subseteq N \backslash i$. A player is null if $v(S \cup i)=v(S)$ for any $S \subseteq N \backslash i$. Remark that a null player is such that $v(i)=0$, hence it is also a dummy player. Note also that the dummy axiom is stronger than the null axiom. The Shapley value [46] is the unique value satisfying axioms $\mathbf{l}, \mathbf{n}, \mathbf{s}$ and efficiency, and is given by

$$
\phi^{v}(i):=\sum_{S \subseteq N \backslash i} \frac{(n-s-1) ! s !}{n !}[v(S \cup i)-v(S)],
$$

where $s:=|S|$.

An equivalent definition can be obtained in a combinatorial way as an average of the contribution of player $i$ over all maximal chains in $2^{N}$ :

$$
\phi^{v}(i)=\frac{1}{n !} \sum_{\mathcal{C} \in \mathcal{M}\left(2^{N}\right)}\left[v\left(S_{\mathcal{C}}^{i}\right)-v\left(S_{\mathcal{C}}^{i} \backslash i\right)\right]
$$

where $\mathcal{M}\left(2^{N}\right)$ is the set of all maximal chains in the lattice $2^{N}$, and for each such chain $\mathcal{C}, S_{\mathcal{C}}^{i}$ is the first subset in the maximal chain containing $i$.

The Shapley value can be also obtained through unanimity games and linearity as follows. Unanimity games are closely linked to the Möbius transform, since any game $v$ can be written as

$$
v=\sum_{S \subseteq N} m(S) u_{S}
$$

where $m$ is the Möbius transform of $v$, and $u_{S}$ is the unanimity game centered on $S$, defined by:

$$
u_{S}(T)= \begin{cases}1, & \text { if } T \supseteq S \\ 0, & \text { else. }\end{cases}
$$

A natural axiom for the Shapley value of unanimity games is

$$
\phi^{u_{S}}(i)= \begin{cases}\frac{1}{|S|}, & \text { if } i \in S \\ 0, & \text { else, }\end{cases}
$$

since only players in $S$ have a contribution to the game, and all players in $S$ are symmetric (anonymous). By linearity of the Shapley value, we get

$$
\phi^{v}(i)=\sum_{S \ni i} \frac{m(S)}{|S|},
$$

which is equivalent to (11). 


\subsection{The Shapley value for multichoice games}

We shall examine in the sequel various propositions for a definition of the Shapley value for multichoice games. We recall that $L=L_{1} \times \cdots \times L_{n}$, and all $L_{i}$ 's are linear lattices, denoted by $L_{i}:=\left\{0,1,2, \ldots, l_{i}\right\}$, where 0 means non participation. Elements $x$ of $L$ are called participation profiles, with $x_{i}$ the level of participation of player $i .\left(0_{-i}, k_{i}\right)$ is the profile where player $i$ plays at level $k$, the other ones not participating. We often write $\widetilde{k_{i}}$ for $\left(0_{-i}, k_{i}\right)$, and $\top_{i}$ for $l_{i}$. Many different approaches exist, which do not coincide:

- approach of Faigle and Kern [17]

- approach of Hsiao and Raghavan 35

- approach of Grabisch and Lange 22, 31

Let us detail first the approach of Faigle and Kern. The basic idea is to axiomatize the Shapley value for unanimity games, and then to apply linearity (combinatorial approach). The expression is the following:

$$
\phi_{\mathrm{FK}}^{v}\left(k_{i}\right)=\frac{1}{|\mathcal{M}(L)|} \sum_{C \in \mathcal{M}(L)}\left[v\left(x_{k_{i}}\right)-v\left(\underline{x}_{k_{i}}\right)\right]
$$

where $\mathcal{M}(L)$ is the set of maximal chains in $L$, and $x_{k_{i}}$ is the first in the sequence $C$ such that $x_{k_{i}} \geq \widetilde{k_{i}}$, and $\underline{x}_{k_{i}}$ is its predecessor. Although the expression is simple and appealing, let us remark that the number of maximal chains for the multichoice case is:

$$
|\mathcal{M}(L)|=\frac{\left(\sum_{i \in N} l_{i}\right) !}{\prod_{i \in N}\left(l_{i} !\right)}=\left(\begin{array}{c}
l \\
l_{1}
\end{array}\right)\left(\begin{array}{c}
l-l_{1} \\
l_{2}
\end{array}\right)\left(\begin{array}{c}
l-l_{1}-l_{2} \\
l_{3}
\end{array}\right) \cdots 1,
$$

with $l:=\prod_{i \in N} l_{i}$. For 5 players having each 3 actions, this already gives $(15) ! / 6^{5}=168,168,000$. Also, some of the axioms proposed by Faigle and Kern are not intuitive in a game theoretic sense (e.g., the hierarchical strength axiom).

The basic idea of the Shapley value of Hsiao and Raghavan is also to axiomatize the Shapley value for unanimity games, and then to apply linearity. The original feature is to put weights $w_{1}<w_{2}<\cdots<w_{l}$ on participation levels. The expression of the Shapley value for unanimity games they obtain is as follows:

$$
\phi_{\mathrm{HR}}^{u_{x}}\left(k_{i}\right)= \begin{cases}\frac{w_{k}}{\sum_{i \in N} w_{x_{i}}}, & \text { if } k=x_{i} \\ 0, & \text { otherwise }\end{cases}
$$

The expression for any game is extremely complex and will not be reported here. It has been proved that for no set of weights $w_{1}, \ldots, w_{l}$, the values of $\mathrm{H}-\mathrm{S}$ and F-K always coincide [6]. Although the axioms are appealing, the resulting formula is almost inapplicable. Also, the role of the weights $w_{1}, \ldots, w_{n}$ is not clear.

We present now our approach. The main idea is to follow as much as possible the original axioms of Shapley. We aim at defining a value $\Phi^{v}\left(k_{i}\right)$ representing the contribution of player $i$ playing at level $k$ vs. non participation of $i$. This contrasts with the two previous approaches, which represent the contribution of player $i$ playing at level $k$ compared to the situation where he plays at level 
$k-1$. For some $k \in L_{i}, k \neq 0$, player $i$ is said to be $k$-null (or simply $k_{i}$ is null) if $v\left(x, k_{i}\right)=v\left(x, 0_{i}\right)$, for any $x \in L_{-i}$. Similarly, for some $k \in L_{i}, k \neq 0$, player $i$ is said to be $k$-dummy (or simply $k_{i}$ is dummy) if $v\left(x, k_{i}\right)=v\left(x, 0_{i}\right)+v\left(\widetilde{k_{i}}\right)$, $\forall x \in L_{-i}$. Based on these definitions, we propose the following axioms.

- Linear axiom (L): $\Phi^{v}$ is linear on the set of games $\mathcal{G}(L)$

- Null axiom (N): $\forall v \in \mathcal{G}(L)$, for all null $k_{i}, \Phi^{v}\left(k_{i}\right)=0$.

- Dummy axiom (D): $\forall v \in \mathcal{G}(L)$, for all dummy $k_{i}, \Phi^{v}\left(k_{i}\right)=v\left(\widetilde{k_{i}}\right)$.

- Monotonicity axiom (M): if $v$ is monotone, then $\Phi^{v}\left(k_{i}\right) \geq 0$, for all $k>0, i \in N$.

The next axiom is similar to the symmetry axiom. Since all lattices $L_{i}$ may be different, a direct transposition of the classical symmetry axiom is not possible. Let $\Gamma(L):=\left\{0_{1}, \top_{1}\right\} \times \cdots \times\left\{0_{n}, \top_{n}\right\}$ be the set of vertices of $L$. We introduce a subspace of $\mathcal{G}(L)$ :

$$
\mathcal{G}_{0}(L):=\{v \in \mathcal{G}(L) \mid v(x)=0, \forall x \notin \Gamma(L)\}
$$

- Symmetry axiom (S): Let $\sigma$ be a permutation on $N$. Then for any game $v \in \mathcal{G}_{0}(L)$,

$$
\Phi^{v^{\sigma^{-1}}}\left(\top_{i}^{\sigma}\right)=\Phi^{v}\left(\top_{i}\right)
$$

where for any $x \in \Gamma(L), x^{\sigma}:=\left(x_{1}^{\sigma}, \ldots, x_{n}^{\sigma}\right)$, and

$$
x_{i}^{\sigma}:= \begin{cases}0_{i}, & \text { if } x_{\sigma(i)}=0_{\sigma(i)} \\ \top_{i}, & \text { if } x_{\sigma(i)}=\top_{\sigma(i)}\end{cases}
$$

and for any $v \in \mathcal{G}_{0}(L), v^{\sigma}$ is a game in $\mathcal{G}_{0}(L)$ such that $v^{\sigma}(x):=v\left(x^{\sigma}\right)$, for any $x \in \Gamma(L)$.

For example, if $L:=\{0,1,2\} \times\{0,1,2,3,4\} \times\{0,1,2,3\}$, and the permutation $\sigma$ is defined by

\begin{tabular}{|c|ccc|}
\hline$i$ & 1 & 2 & 3 \\
$\sigma(i)$ & 2 & 3 & 1 \\
\hline
\end{tabular}

then $(2,0,0)^{\sigma}=(0,0,3),(2,0,3)^{\sigma}=(0,4,3)$.

Next axiom is not in the original set of axioms of Shapley, and concerns a kind of homogeneity of the structure of the $L_{i}$ 's.

- Invariance axiom (I): Let us consider $v_{1}, v_{2}$ on $L$ such that for some $i \in N$,

$$
\begin{aligned}
& v_{1}\left(x, k_{i}\right)=v_{2}\left(x,(k-1)_{i}\right), \quad \forall x \in L_{-i}, \forall 1<k \leq l_{i} \\
& v_{1}\left(x, 0_{i}\right)=v_{2}\left(x, 0_{i}\right), \quad \forall x \in L_{-i} .
\end{aligned}
$$

Then $\Phi^{v_{1}}\left(k_{i}\right)=\Phi^{v_{2}}\left((k-1)_{i}\right), 1<k \leq l_{i}$.

- Efficiency axiom (E): $\sum_{i \in N} \Phi^{v}\left(\top_{i}\right)=v(\top)$. 
Proposition 2 Under axioms $(\boldsymbol{L}),(\boldsymbol{D}),(\boldsymbol{M}),(\boldsymbol{S}),(\boldsymbol{I})$ and $(\boldsymbol{E})$,

$$
\Phi^{v}\left(k_{i}\right)=\sum_{x \in \Gamma\left(L_{-i}\right)} \frac{(n-h(x)-1) ! h(x) !}{n !}\left[v\left(x, k_{i}\right)-v\left(x, 0_{i}\right)\right], \quad 1 \leq k \leq l_{i}, i \in N,
$$

where $h(x):=\left|\left\{k \in N \backslash i \mid x_{k}=\top_{k}\right\}\right|$.

Remark that the result is very close to the classical formula of Shapley. For a formula on more general lattices (but without axiomatization) and for the interaction index, see 29].

\section{The core}

\subsection{The classical case}

In game theory, the core of $v$ is another way to define rational imputations for players. Specifically, it is a set of imputations such that no subcoalition has interest to form:

$$
\mathcal{C}(v):=\left\{\phi \in \mathbb{R}^{n} \mid \phi(N)=v(N) \text { and } \phi(A) \geq v(A), \forall A \subseteq N\right\}
$$

with $\phi(A):=\sum_{i \in A} \phi(i)$. Otherwise said, it is the set of additive games dominating $v$ and coinciding on $N$. Whenever nonempty, the core is a convex set. It is reduced to the singleton $\{v\}$ if the game is additive.

The same concept exists also in capacity theory. It is seen as the set of probability measures dominating a given capacity (see properties of the core in [9]).

A related concept is the Weber set. It is the convex hull of the set $\mathcal{M}(v)$ of marginal worth vectors

$$
\mathcal{W}(v):=\operatorname{co}(\mathcal{M}(v))
$$

where a marginal worth vector is defined as the increment of $v$ along a maximal chain in the Boolean lattice $2^{N}$. Specifically, to any permutation $\pi$ on $N$, we associate a maximal chain

$$
A_{0}^{\pi}:=\emptyset \subset A_{1}^{\pi}:=\{\pi(1)\} \subset A_{2}^{\pi}:=\{\pi(1), \pi(2)\} \subset \cdots \subset A_{n}^{\pi}:=N
$$

with $A_{i}^{\pi}:=\{\pi(1), \ldots, \pi(i)\}$. Then, the corresponding marginal worth vector $x^{\pi}(v)$ is defined by:

$$
x_{\pi(i)}^{\pi}(v):=v\left(A_{i}^{\pi}\right)-v\left(A_{i-1}^{\pi}\right), \quad i=1, \ldots, n .
$$

The following proposition summarizes well-known results. We recall that a game is convex if $v(A \cup B)+v(A \cap B) \geq v(A)+v(B)$ for any $A, B \subseteq N$.

Proposition 3 Let $v$ be a game on $N$. The following holds.

(i) $\mathcal{C}(v) \subseteq \mathcal{W}(v)$

(ii) $\mathcal{C}(v) \neq \emptyset$ if $v$ is convex.

(iii) $v$ is convex iff $\mathcal{C}(v)=\mathcal{W}(v)$ (i.e., the set of marginal worth vectors is the set of vertices of the core). 


\subsection{The case of multichoice games}

Here also, several different approaches have been proposed, the first one being by Faigle [16], see also the works of Tijs et al. [51]. For a detailed comparison of these previous works with our approach, see [53, 32]. We give below the main elements of our approach, and the one of Faigle.

$v$ being a multichoice game, we say that $v$ is convex if $v(x \vee y)+v(x \wedge y) \geq$ $v(x)+v(y)$, for all $x, y \in L$, and $v$ is additive if for every $x, y \in L$ such that $x \wedge y=\perp$, it holds $v(x \vee y)=v(x)+v(y)$.

We denote by $\mathcal{A}(L)$ the set of additive games on $L$. The following definition is a direct transposition of the classical definition.

Definition 6 The precore of a multichoice game $v$ on $L$ is defined by

$$
\mathcal{P C}(v):=\{\phi \in \mathcal{A}(L) \mid \phi(x) \geq v(x), \forall x \in L, \text { and } \phi(\top)=v(\top)\} .
$$

This is in fact the definition of Faigle. However, it is easy to see that the precore, although convex, is unbounded. Indeed, considering a 2-choice game with two players, hence $L:=\{0,1,2\}^{2}$, the conditions on $\phi$ to be element of the precore write:

$$
\begin{aligned}
\phi(2,0)+\phi(0,2) & =v(2,2) \\
\phi(1,0) & \geq v(1,0) \\
\phi(0,1) & \geq v(0,1) \\
\phi(1,0)+\phi(0,1) & \geq v(1,1) .
\end{aligned}
$$

Remark that $\phi(1,0)$ and $\phi(0,1)$ may be taken arbitrarily large. We denote by $\mathcal{P C}^{F}(v):=\operatorname{co}(\operatorname{Ext}(\mathcal{P C}(v)))$ the polytope of $\mathcal{P C}(v)$, where $\operatorname{Ext}()$ is the set of extreme points (vertices) of some convex set.

To avoid these drawbacks, we propose the next definition, where normalization occurs at each level.

Definition 7 The core of a multichoice game $v$ on $N$ is defined as:

$$
\begin{aligned}
\mathcal{C}(v):= & \{\phi \in \mathcal{A}(L) \mid \phi(x) \geq v(x), \forall x \in L \\
& \text { and } \left.\phi\left(k \wedge l_{1}, \ldots, k \wedge l_{n}\right)=v\left(k \wedge l_{1}, \ldots, k \wedge l_{n}\right), k=1, \ldots, \max _{j} l_{j}\right\} .
\end{aligned}
$$

As for the classical case, we introduce marginal worth vectors $\psi^{C}$ as the vectors of increments along maximal chains $C$ in the lattice $L$. Coordinates of $\psi^{C}$ are denoted by $\psi_{k_{j}}^{C}$, for any player $j \in N$ and any level $k>0$ in $L_{j}$. To any marginal vector is associated an additive game $\phi^{C}$ defined by

$$
\phi_{k_{j}}^{C}:=\sum_{p=1}^{k} \psi_{p_{j}}^{C} .
$$

The set of all such additive games is called $\mathcal{P} \mathcal{M}(v)$, and the pre-Weber set $\mathcal{P} \mathcal{W}(v)$ is defined as the convex hull of all additive games in $\mathcal{P} \mathcal{M}(v)$. Considering only restricted maximal chains in $L$, i.e., those passing through all $\left(k \wedge l_{1}, \ldots, k \wedge l_{n}\right), k=1, \ldots, \max _{j} l_{j}$, we define $\mathcal{M}(v)$, the set of all additive games $\phi^{C}$ corresponding to marginal worth vectors associated to all restricted 
maximal chains. Then the Weber set $\mathcal{W}$ is defined as the convex hull of all additive games in $\mathcal{M}(v)$. 田.

The following has been shown, which generalizes the classical results of Prop.

Proposition 4 Let $v$ be a multichoice games on L. The following holds.

(i) $\mathcal{P C}^{F}(v) \subseteq \mathcal{P} \mathcal{W}(v)$

(ii) $\mathcal{C}(v) \subseteq \mathcal{W}(v)$

(iii) If $v$ is convex, then $\mathcal{C}(v)=\mathcal{W}(v)$

(iv) If $v$ is convex, then $\mathcal{P C}^{F}(v)=\mathcal{P W}(v)$.

\section{The entropy}

The entropy is a central notion in probability and information theory. The first attempt to generalize the classical definition of Shannon to the case of capacities was done by Yager [54], by considering the Shannon entropy of the Shapley value of the capacity. A slightly different approach was taken by Marichal and Roubens [43, 42], which turned out to have better properties. In particular, it is strictly increasing towards the capacity which maximizes entropy. Its expression for some capacity $\mu$ is given below:

$$
H_{\mathrm{MR}}(\mu):=\sum_{i=1}^{n} \sum_{S \subseteq N \backslash i} \frac{(n-s-1) ! s !}{n !} h(\mu(S \cup i)-\mu(S))
$$

where $h(x):=-x \log x$, for $x>0$, and $h(0):=0$. An important result, due to Dukhovny [15], shows that the definition of Marichal and Roubens can be written as an average of classical entropy along maximal chains of the Boolean lattice $2^{N}$, specifically:

$$
H_{\mathrm{MR}}(\mu)=\frac{1}{n !} \sum_{\mathcal{C} \in \mathcal{M}\left(2^{N}\right)} H_{\mathrm{S}}\left(p^{\mu, \mathcal{C}}\right)
$$

where $H_{\mathrm{S}}$ is the Shannon entropy, and $p^{\mu, \mathcal{C}}$ the probability induced by the maximal chain $\mathcal{C}$ and the capacity $\mu$, i.e., using the same notations as for Eq. (12):

$$
p^{\mu, \mathcal{C}}(\{i\})=\mu\left(S_{\mathcal{C}}^{i}\right)-\mu\left(S_{\mathcal{C}}^{i} \backslash i\right), \quad i \in N
$$

(identical to marginal worth vectors).

Honda and Grabisch have shown that the above definition could be generalized without losing its nice properties for capacities on particular set systems [34. Let us consider $\mathcal{N}$ a subcollection of $2^{N}$. Then we call $(N, \mathcal{N}$ ) (or simply $\mathcal{N}$ if no ambiguity occurs) a set system if $\mathcal{N}$ contains $\emptyset$ and $N$. A set system is a particular partially ordered set when endowed with inclusion, hence usual definitions apply, in particular the notion of maximal chain. We denote the set of all maximal chains of $\mathcal{N}$ by $\mathcal{M}(\mathcal{N}) .(N, \mathcal{N})$ is a regular set system if for any $\mathcal{C} \in \mathcal{M}(\mathcal{N})$, the length of $\mathcal{C}$ is $n$, i.e. $|\mathcal{C}|=n+1$. Equivalently, $\mathcal{N}$ is a regular set system if and only if $|A \backslash B|=1$ for any $A, B \in \mathcal{N}$ such that $A \succ B$. Let $\mu$ 
be a capacity on $(N, \mathcal{N})$. For any $\mathcal{C} \in \mathcal{M}\left(2^{N}\right)$, define $p^{\mu, \mathcal{C}}$ by (32) again. Then the entropy of $\mu$ on $(N, \mathcal{N})$ is given by:

$$
H_{\mathrm{HG}}(\mu):=\frac{1}{|\mathcal{M}(\mathcal{N})|} \sum_{\mathcal{C} \in \mathcal{M}(\mathcal{N})} H_{S}\left(p^{\mu, \mathcal{C}}\right) .
$$

$H_{\mathrm{HG}}$ is a continuous function of $\mu$, and $0 \leq H_{\mathrm{HG}} \leq \log n$, with equality at left attained if and only if $\mu$ is a 0-1 valued capacity, and at right if and only if $\mu$ is the additive uniform capacity. Moreover, $H_{\mathrm{HG}}$ is strictly increasing towards the value of the additive uniform capacity.

The entropy for capacities has been axiomatized by Kojadinovic et al. 37 using a recursive axiom difficult to interpret. Honda and Grabisch have axiomatized $H_{\mathrm{HG}}$ in a different way [33], avoiding such an axiom, and following Faddeev's classical axiomatization of Shannon entropy.

\section{The case of bipolar structures}

Let us come back on bi-capacities and bi-cooperative games. First works on bicapacities [23, 25, 26] have taken for granted that these were capacities defined on the lattice $(\mathcal{Q}(N)$, $)$, with $\left(A, A^{\prime}\right) \sqsubseteq\left(B, B^{\prime}\right) \Leftrightarrow A \subseteq B$ and $A^{\prime} \supseteq B^{\prime}$. Doing so, bi-capacities are indeed monotonic mappings and match the general

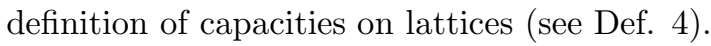

There is nevertheless something discordant in the fact that doing so, since $(\mathcal{Q}(N), \sqsubseteq)$ is isomorphic to the lattice $3^{n}$, bi-cooperative games become in some sense isomorphic to multichoice games with $m=2$, a conclusion which may be surprising if one consider the interpretation behind them. Let us elaborate on this last point. We may say that for a 2-choice game, the underlying levels of participation are naturally labelled $0,1,2$, with 0 indicating non participation, 1 a mild participation, and 2 a full participation. For bi-cooperative games, keeping the same labelling leads to something rather odd, since 0 means (full) participation against, 1 non participation, and 2 (full) participation. Hence, a more natural labelling would be $-1,0,1$, the 0 value being central, and $-1,1$ being symmetric extremes. This suggests that:

(i) the point $(\emptyset, \emptyset)$ is central in the structure $\mathcal{Q}(N)$, although in $3^{N},(1, \ldots, 1)$ has no central role;

(ii) bi-cooperative games are not 2-choice games, but rather a symmetrization of classical cooperative games.

Looking at the definition of the Choquet integral for bi-capacities (Sec. 5), one can see that it already follows the above principle.

Consequently, the order $\sqsubseteq$ should be replaced by the product order $\subseteq$ : $\left(A, A^{\prime}\right) \subseteq\left(B, B^{\prime}\right) \Leftrightarrow A \subseteq B A^{\prime} \subseteq B^{\prime}$. Interestingly enough, this was the first definition proposed by Bilbao [3] for the underlying structure of bi-cooperative games. Now, $(\mathcal{Q}(N), \subseteq)$ is no more a lattice, but an inf-semilattice.

Consequently, a proper definition of the Möbius transform is not the one proposed in [25], solution of the equation:

$$
v\left(A, A^{\prime}\right)=\sum_{\left(B, B^{\prime}\right) \sqsubseteq\left(A, A^{\prime}\right)} m\left(B, B^{\prime}\right)
$$


but it should be the solution of the equation:

$$
v\left(A, A^{\prime}\right)=\sum_{\left(B, B^{\prime}\right) \subseteq\left(A, A^{\prime}\right)} m\left(B, B^{\prime}\right)
$$

whose solution is:

$$
m\left(A, A^{\prime}\right)=\sum_{\substack{B \subseteq \subseteq \\ B^{\prime} \subseteq A^{\prime}}}(-1)^{|A \backslash B|+\left|A^{\prime} \backslash B^{\prime}\right|} v\left(B, B^{\prime}\right) .
$$

This function $m$, which could be called the bipolar Möbius transform, has been first proposed by Fujimoto [19, 20], in order to avoid the complicated expression of the Choquet integral in terms of the Möbius transform given in [26]. Indeed, using the (bipolar) Möbius transform, the Choquet integral simply writes:

$$
\int f d v=\sum_{\left(A, A^{\prime}\right) \in \mathcal{Q}(N)} m\left(A, A^{\prime}\right)\left[\bigwedge_{i \in A} f_{i}^{+} \wedge \bigwedge_{j \in A^{\prime}} f_{j}^{-}\right] .
$$

The definition of entropy for bi-capacities, as it is given by Kojadinovic and Marichal [36], follows in fact the same philosophy. It writes:

$$
H_{\mathrm{KM}}(v):=\frac{1}{2^{n}} \sum_{N^{+} \subseteq N} \frac{1}{n !} \sum_{\pi \in \Pi_{N}} H_{S}\left(p_{\pi, N^{+}}^{v}\right)
$$

where $\pi$ is any permutation on $N$, and $p_{\pi, N^{+}}^{v}$ is the probability distribution induced by $v$ and the maximal chain induced by $\pi$ in the sublattice $\left[(\emptyset, \emptyset),\left(N^{+}, N \backslash\right.\right.$ $\left.N^{+}\right)$.

In summary, bi-capacities and bi-cooperative games should be considered as a particular symmetrization of capacities and cooperative games, as well as $\mathcal{Q}(N)$ should be considered as a symmetrization of $\mathcal{P}(N)=2^{N}$. We call this particular symmetrization bipolar extension, and show now that this can be made fairly more general [28].

Definition 8 Let $(L, \leq)$ be an inf-semilattice with bottom element $\perp$. We define its bipolar extension by

$$
\widetilde{L}:=\{(x, y) \mid x, y \in L, x \wedge y=\perp\},
$$

which we endow with the product order $\leq$ on $L^{2}$.

Clearly, $\mathcal{Q}(N)=\widetilde{\mathcal{P}(N)}$. The following holds.

Proposition 5 Let $(L, \leq)$ be an inf-semilattice.

(i) $(\widetilde{L}, \leq)$ is an inf-semilattice whose bottom element is $(\perp, \perp)$, where $\leq$ is the product order on $L^{2}$.

(ii) The set of join-irreducible elements of $\widetilde{L}$ is

$$
\mathcal{J}(\widetilde{L})=\{(j, \perp) \mid j \in \mathcal{J}(L)\} \cup\{(\perp, j) \mid j \in \mathcal{J}(L)\} .
$$

(iii) The Möbius function on $\widetilde{L}$ is given by:

$$
\mu_{\tilde{L}}((z, t),(x, y))=\mu_{L}(z, x) \mu_{L}(t, y) .
$$

Bipolar extensions have been further investigated in [27], concerning the definition of the Choquet integral or other aggregation operators on such structures. 


\section{Concluding remarks}

The above compilation of results shows that the field is quite active, and seems to be promising. Future directions of research are clearly in the direction of set systems, in particular what is called regular set systems [34] (see Section 9). A set system is regular if all its maximal chains are of same length. It can be shown that these structures encompass distributive lattices and convex geometries, and are therefore fairly general. With regular set systems, all the usual tools defined here (Choquet integral, Shapley value, entropy, core) can be defined since all these notions can be defined through maximal chains.

\section{References}

[1] J.-P. Aubin. Cooperative fuzzy games. Mathematics of Operations Research, 6:1-13, 1981.

[2] J. M. Bilbao. Axioms for the Shapley value on convex geometries. European Journal of Operational Research, 110:368-376, 1998.

[3] J. M. Bilbao. Cooperative games on combinatorial structures. Kluwer Academic Publishers, 2000.

[4] J. M. Bilbao, E. Lebrón, and N. Jiménez. The core of games on convex geometries. European Journal of Operational Research, 119:365-372, 1999.

[5] G. Birkhoff. Lattice Theory. American Mathematical Society, 3d edition, 1967.

[6] R. Branzei, D. Dimitrov, and S. Tijs. Models in cooperative game theory: crisp, fuzzy and multichoice games. Springer Verlag, 2005.

[7] D. Butnariu and E. P. Klement. Triangular norm-based measures and games with fuzzy coalitions. Kluwer Academic Publishers, 1993.

[8] H. Cartan. Théorie du potentiel newtonien : energie, capacité, suites de potentiels. Bulletin de la Société Mathématique de France, 73:74-106, 1945.

[9] A. Chateauneuf and J.-Y. Jaffray. Some characterizations of lower probabilities and other monotone capacities through the use of Möbius inversion. Mathematical Social Sciences, 17:263-283, 1989.

[10] G. Choquet. Theory of capacities. Annales de l'Institut Fourier, 5:131-295, 1953.

[11] I. Curiel. Cooperative Game Theory and Applications. Kluwer Academic Publ., 1997.

[12] B. A. Davey and H. A. Priestley. Introduction to Lattices and Orders. Cambridge University Press, 1990.

[13] D. Denneberg. Non-Additive Measure and Integral. Kluwer Academic, 1994. 
[14] T. Driessen. Cooperative Games, Solutions and Applications. Kluwer Academic Publishers, 1988.

[15] A. Dukhovny. General entropy of general measures. Int. J. Uncertainty, Fuzziness and Knowledge-Based Systems, 10:213-225, 2002.

[16] U. Faigle. Cores with restricted cooperation. ZOR - Methods and Models of Operations Research, pages 405-422, 1989.

[17] U. Faigle and W. Kern. The Shapley value for cooperative games under precedence constraints. Int. J. of Game Theory, 21:249-266, 1992.

[18] D. Felsenthal and M. Machover. Ternary voting games. Int. J. of Game Theory, 26:335-351, 1997.

[19] K. Fujimoto. New characterizations of $k$-additivity and $k$-monotonicity of bi-capacities. In SCIS-ISIS 2004, 2nd Int. Conf. on Soft Computing and Intelligent Systems and 5th Int. Symp. on Advanced Intelligent Systems, Yokohama, Japan, September 2004.

[20] K. Fujimoto. Some characterizations of $k$-monotonicity through the bipolar möbius transform in bi-capacities. J. of Advanced Computational Intelligence and Intelligent Informatics, 9(5):484-495, 2005.

[21] I. Gilboa and E. Lehrer. Global games. Int. J. of Game Theory, 20:129-147, 1991.

[22] M. Grabisch. An axiomatization of the Shapley value and interaction index for games on lattices. In SCIS-ISIS 2004, 2nd Int. Conf. on Soft Computing and Intelligent Systems and 5th Int. Symp. on Advanced Intelligent Systems, Yokohama, Japan, September 2004.

[23] M. Grabisch and Ch. Labreuche. Bi-capacities for decision making on bipolar scales. In EUROFUSE Workshop on Informations Systems, pages 185190, Varenna, Italy, September 2002.

[24] M. Grabisch and Ch. Labreuche. Capacities on lattices and $k$-ary capacities. In 3d Int, Conf. of the European Soc. for Fuzzy Logic and Technology (EUSFLAT 2003), pages 304-307, Zittau, Germany, September 2003.

[25] M. Grabisch and Ch. Labreuche. Bi-capacities. Part I: definition, Möbius transform and interaction. Fuzzy Sets and Systems, 151:211-236, 2005.

[26] M. Grabisch and Ch. Labreuche. Bi-capacities. Part II: the Choquet integral. Fuzzy Sets and Systems, 151:237-259, 2005.

[27] M. Grabisch and Ch. Labreuche. A general construction for unipolar and bipolar interpolative aggregation. In 4th Conf. of the Eur. Soc. for Fuzzy Logic And Technology (EUSFLAT) and 11th LFA Conf., pages 916-921, Barcelona, Spain, Sept. 2005.

[28] M. Grabisch and Ch. Labreuche. Bi-capacities: towards a generalization of Cumulative Prospect Theory. J. of Mathematical Psychology, submitted. 
[29] M. Grabisch and Ch. Labreuche. Derivative of functions over lattices as a basis for the notion of interaction between attributes. Discrete Applied Maths., to appear.

[30] M. Grabisch, Ch. Labreuche, and J.-C. Vansnick. On the extension of pseudo-Boolean functions for the aggregation of interacting bipolar criteria. Eur. J. of Operational Research, 148:28-47, 2003.

[31] M. Grabisch and F. Lange. Games on lattices, multichoice games and the Shapley value: a new approach. Mathematical Methods of Operations Research, to appear.

[32] M. Grabisch and L. J. Xie. A new investigation about the core and Weber set of multichoice games. Mathematical Methods of Operations Research, submitted.

[33] A. Honda and M. Grabisch. An axiomatization of entropy of capacities on set systems. Eur. J. of Operational Research, submitted.

[34] A. Honda and M. Grabisch. Entropy of capacities on lattices and set systems. Information Sciences, to appear.

[35] C. R. Hsiao and T. E. S. Raghavan. Shapley value for multichoice cooperative games, I. Games and Economic Behavior, 5:240-256, 1993.

[36] I. Kojadinovic and J.-L. Marichal. Entropy of bi-capacities. In 4th Conf. of the Eur. Soc. for Fuzzy Logic And Technology (EUSFLAT) and 11th LFA Conf., pages 991-996, Barcelona, Spain, September 2005.

[37] I. Kojadinovic, J.-L. Marichal, and M. Roubens. An axiomatic approach to the definition of the entropy of a discrete Choquet capacity. Information Sciences, 172:131-153, 2005.

[38] B. Korte, L. Lovász, and R. Schrader. Greedoids. Springer Verlag, 1991.

[39] Ch. Labreuche and M. Grabisch. The Choquet integral for the aggregation of interval scales in multicriteria decision making. Fuzzy Sets and Systems, 137:11-26, 2003.

[40] L. Lovász. Submodular function and convexity. In A. Bachem, M. Grötschel, and B. Korte, editors, Mathematical programming. The state of the art, pages 235-257. Springer Verlag, 1983.

[41] J.-L. Marichal. Aggregation of interacting criteria by means of the discrete Choquet integral. In T. Calvo, G. Mayor, and R. Mesiar, editors, Aggregation operators: new trends and applications, volume 97 of Studies in Fuzziness and Soft Computing, pages 224-244. Physica Verlag, 2002.

[42] J.-L. Marichal. Entropy of discrete Choquet capacities. Eur. J. of Oper. Res., 137:612-624, 2002.

[43] J.-L. Marichal and M. Roubens. Entropy of discrete fuzzy measures. Int. J. Uncertainty, Fuzziness and Knowledge-Based Systems, 8:625-640, 2000. 
[44] B. Peleg and P. Sudhölter. Introduction to the theory of cooperative games. Kluwer Academic Publisher, 2003.

[45] G. C. Rota. On the foundations of combinatorial theory I. Theory of Möbius functions. Zeitschrift für Wahrscheinlichkeitstheorie und Verwandte Gebiete, 2:340-368, 1964.

[46] L. S. Shapley. A value for $n$-person games. In H. W. Kuhn and A. W. Tucker, editors, Contributions to the Theory of Games, Vol. II, number 28 in Annals of Mathematics Studies, pages 307-317. Princeton University Press, 1953.

[47] I. Singer. Extensions of functions of 0-1 variables and applications to combinatorial optimization. Numerical Functional Analysis and Optimization, $7(1): 23-62,1984$.

[48] M. Sugeno. Theory of fuzzy integrals and its applications. PhD thesis, Tokyo Institute of Technology, 1974.

[49] R. M. Thrall and W. F. Lucas. n-person games in partition function form. Naval Research Logistic Quarterly, 10:281-298, 1963.

[50] S. Tijs, R. Branzei, S. Ishihara, and S. Muto. On cores and stable sets for fuzzy games. Fuzzy Sets and Systems, 146:285-296, 2004.

[51] A. van den Nouweland, S. Tijs, J. Potters, and J. Zarzuelo. Cores and related solution concepts for multi-choice games. ZOR - Mathematical Methods of Operations Research, 41:289-311, 1995.

[52] F. Vasilesco. La notion de capacité. Hermann, Paris, 1937.

[53] L. J. Xie and M. Grabisch. The core of capacities on lattices. In Proc. of the Int. Fuzzy Systems Association World Congress (IFSA), pages 461-466, Beijing, China, July 2005.

[54] R. R. Yager. On the entropy of fuzzy measures. IEEE TR. on Fuzzy Systems, 8:453-461, 2000. 